\title{
Zehn Herausforderungen im öffentlichen Gesundheitswesen
}

\author{
Public Health ist wie die Politik die Kunst des Möglichen, sagt \\ der Direktor des US-Centers for Disease Control und Preven- \\ tion (CDC), Thomas R. Frieden. Er fasst in 10 Punkten zusam- \\ men, was die im öffentlichen Gesundheitswesen Tätigen alles \\ für eine bessere Gesundheitsförderung tun können. \\ Frieden TR et al. Ten Things I Wish Someone Had Told Me \\ When I Became a Health Officer. Am J Publ Health 2016; 106: \\ 1214-1218
}

Thomas R. Frieden meint, Angestellte und Entscheidungsträger im öffentlichen Gesundheitswesen sollten die Wirksamkeit ihrer Tätigkeit wie folgt verbessern:

\section{Besorgen und verbreiten Sie gute Daten \\ $\nabla$}

Sorgen Sie für eine gute Datengrundlage, z.B. zu einem nicht gedeckten Bedarf im Gesundheitswesen oder zu wichtigen Ressourcen, und verbreiten Sie diese Daten zeitnah unter allen, die Sie für Entscheidungen und die Projektsteuerung benötigen. Wenn gute Daten aus zugänglichen Quellen nicht verfügbar sind, sollten Ressourcen auf die wichtigsten Fragestellungen fokussiert werden, um gezielt gute Daten zu erheben.

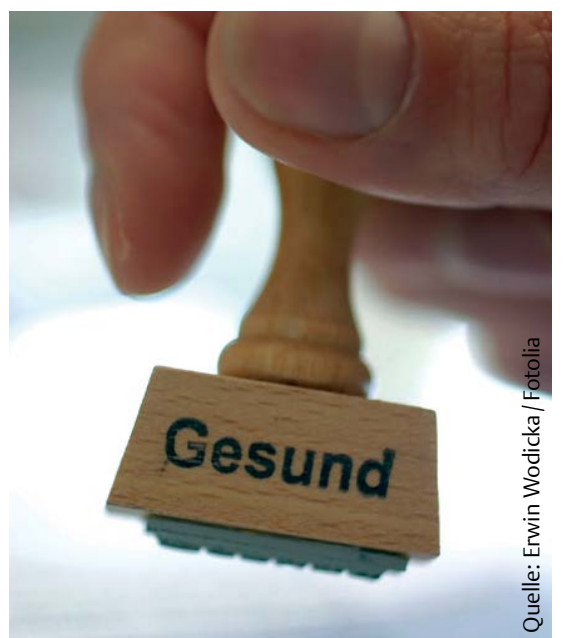

Für ein "gesundes" öffentliches Gesundheitssystem kann und sollte jeder Akteur mehr tun, meint Thomas R. Frieden - und gibt konkrete Anregungen.
2. Priorisieren Sie und gehen Sie schwierige Aktivitäten zuerst an $\nabla$

Im Bereich Public Health muss ständig zwischen verschiedenen Gesundheitszielen priorisiert und dabei im Auge gehalten werden, was möglich ist. Es ist verführerisch, zunächst die Dinge anzugehen, die leicht zu erreichen scheinen. Besser ist allerdings, wichtige, aber schwierige und möglicherweise kontroverse Initiativen primär zu verfolgen.

\section{Finden, kämpfen und gewinnen Sie Kämpfe, die gewinnbar sind $\nabla$}

Dem steht nicht entgegen, auch immer mindestens ein gut erreichbares Ziel zu verfolgen, dass einen Fortschritt zum Schutz und der Verbesserung der Gesundheit darstellt und auch öffentlich wahrnehmbaren Erfolg verspricht. Es ist daher wichtig, Bereiche zu identifizieren, in denen solch ein Fortschritt möglich ist, aber bisher wegen fehlender Fokussierung auf ein Ziel noch nicht erreicht wurde.

\section{Unterstützten Sie gute Leute} und schützen Sie sie, so dass sie ihre Arbeit tun können $\nabla$

Erfolg ist nur mit Mitarbeitern möglich, die in einem weiten Spektrum von PublicHealth-Bereichen sowohl inhaltliche als auch operative Stärken mitbringen. Solche Menschen einzustellen, weiterzuentwickeln, zu halten und ihnen Verantwortung zu übertragen, ist extrem wichtig. Sie benötigen aber auch - wo nötig - den Schutz z.B. vor politischem Druck.
5. Gehen Sie die Themen übertragbare Krankheiten und Umwelteinflüsse auf die Gesundheit effektiv an $\nabla$

Diese Themen sind und bleiben Kernthemen des öffentlichen Gesundheitswesens. Sollte ein im Gesundheitswesen Verantwortlicher einen Ausbruch einer Infektionswelle oder eine gesundheitliche Gefahr aus der Umwelt nicht schnell genug identifizieren und mit sensiblen und wirksamen Maßnahmen eindämmen, wird dies Presse, Öffentlichkeit und die eigenen Mitarbeiter für einige Zeit so sehr beschäftigen, dass andere wichtige $\mathrm{Ge}$ sundheitsziele auf der Strecke bleiben.

6. Geben Sie die Kooperation zwischen klinischem Bereich und öffentl. Gesundheitswesen nicht auf $\nabla$

Die klinische Versorgung in all ihren Facetten zu verstehen und zu verbessern, ist für die meisten Ziele im öffentlichen Gesundheitswesen essenziell. Diagnose, Berichterstattung und Therapie sind wesentliche Komponenten bei der Lösung von Public-Health-Problemen im Bereich von Infektionskrankheiten, Prävention oder Impfung. Die Zusammenarbeit mit Ärzten, Kliniken, Fachorganisationen, Krankenkassen, Arbeitgebern und anderen ist darüber hinaus wesentlich für eine bessere Versorgung chronisch Kranker. Eine Aufgabenteilung im Sinne einer Kooperation kann helfen, öffentliche Gesundheitsziele zu erreichen.

\section{Verstehen und bewältigen Sie den Budgetzyklus} $\nabla$

Budgets folgen einem saisonalen Zyklus. Ein Verantwortlicher im Bereich Public Health sollte sich immer vergegenwärtigen, wo in seinem Verantwortungsbereich Einschnitte ohne zu großen Schaden möglich sein könnten und wo andererseits zusätzliche Budgets sinnvoll und nutzbringend eingesetzt werden könnten. Bei der Chance auf frei werdende Budgets sollte man Programme vorstellen können, in die neu bereitgestellte Mittel mit einem größtmöglichen Gewinn für die Gesundheit fließen können.

\section{Managen Sie auch das Umfeld 7}

Die Öffentlichkeit bestimmt mit die Freiheit, tatsächlich etwas in der Gesund- 
heitsversorgung bewegen zu können. Deshalb ist eine gute Öffentlichkeitsarbeit z. B. bei einem Notfall wichtig, ein Medientraining empfehlenswert.

\section{9. Überraschen Sie nicht lhren \\ Chef \\ $\nabla$}

Wenn sich etwas entwickelt, insbesondere etwas Negatives, sollte das Ihr Vorgesetzter direkt und frühzeitig von Ihnen erfahren, nicht zuerst aus den Medien. Sind Sie direkt einem gewählten Vertreter unterstellt, muss sich dieser auf Sie in Fachfragen oder organisatorischen Details verlassen können - so wie Sie seine Entscheidungshoheit akzeptieren müssen und sich vergegenwärtigen sollten, welchem öffentlichen Druck er ausgesetzt ist. Er braucht die frühe Warnung, wenn etwas vor sich geht.

\section{Bleiben Sie den 5 Kernprinzipien von Dowdle treu \\ $\nabla$}

Die 5 Kernprinzipien, die Walter Dowdle als Direktor des CDC formulierte, sind:

1. Seien Sie ein gewissenhafter Verwalter der Mittel, die Ihnen anvertraut wurden.

2. Schaffen Sie ein Arbeitsumfeld, das Raum lässt für intellektuelles und persönliches Wachstum und Integrität.

3. Begründen Sie alle Ihre Entscheidungen auf einer größtmöglichen Qualität wissenschaftlicher Daten, die transparent und objektiv ermittelt wurden.

4. Setzen Sie den Gewinn für die Gesellschaft über den Gewinn Ihrer Einrichtung oder Organisation.

5. Behandeln Sie alle Menschen mit Würde, Aufrichtigkeit und Respekt.

\section{Fazit}

Thomas R. Frieden betont, jeder Angestellte im öffentlichen Gesundheitswesen hat das Privileg und die Verantwortung, den Staffelstab des gesundheitlichen Fortschritts zu übernehmen und so schnell und weit wie möglich im Sinne eines gesunden und langen Lebens für die Menschen weiterzutragen. Und diesen Stab schließlich so gut wie möglich an andere weiterzugeben, die diesen Fortschritt zukünftig voranbringen. 